\title{
ETHICAL ISSUES IN THE USE OF TECHNOLOGY IN CLINICAL SUPERVISION
}

\author{
Katja Belšak, Anja Simonič
}

\begin{abstract}
The article explores ethical issues raised by the use of technology in clinical work with clients and in the supervision of supervisees' work with clients. In the first part, the new technologies are presented, as well as the benefits and drawbacks of their use in supervision. The literature reveals three fields in which ethical issues are raised: supervision process, legal and regulatory issues and technology. In more detail, major emphasis has been put on the supervisory working relationship and questions of process, informed consent (between a supervisor and a supervisee and between a supervisee and a client), evaluation of a supervisees' efficacy and confidentiality.
\end{abstract}

\section{KEY WORDS}

supervision, new technologies, ethical issues, informed consent

Katja Belšak, clinical psychologist, MSc

Anja Simonič, clinical psychologist, $\mathrm{PhD}$

\section{INTRODUCTION}

In the last decades, there has been an increasing focus on the use of technology in everyday clinical work which includes psychotherapy and supervision. In comparison to 20 years ago, the resources available now are amazing if we look at the evolution of technology in Table 1 (1). In psychotherapy training and supervision, we now use e-mails, text messaging, video-conferences (Skype), phone supervision, digital recording technology, internet clouds, etc.
The primary role of clinical supervisors is to serve as professional gatekeepers: to continually monitor and evaluate their supervisees' clinical practice as a way of protecting and safeguarding the well-being of clients (2). Technology has helped supervisors gather much more information about supervisees and clients than ever before. Bernard et al. (2) identify a number of benefits, including a decreased amount of time lost to travel, convenience in scheduling, larger pool 
Table 1. Evolution of technology (1).

\begin{tabular}{|l|l|}
\hline Pre-2000 & Post-2000 \\
\hline Devices are mechanical & Devices run on software \\
\hline Devices are standalone & Devices connect via networks and the Internet \\
\hline Devices are designed to save data & Devices are designed to share data \\
\hline Devices turn on/off & Devices are designed to be always on \\
\hline Devices are static & Devices constantly update themselves \\
\hline Devices serve only the user & Devices can serve the user manufacturer, or others \\
\hline Devices are single purpose & Devices are multifunctional \\
\hline Data is stored locally & Data can be stored in multiple distant locations \\
\hline Pre-2000 & Post-2000 \\
\hline
\end{tabular}

of available supervisors, quick and easy access to records and paperwork (also for reviewing), limited time constraints and geographic challenges, low cost, increased access to multicultural supervision and leaving an electronic trail of communication (e.g. emails, saved documents, file retrieval).

On the other hand, the drawbacks of the use of technology are as follows: at times, supervisors are unable to keep up with changes in technology, and they may feel uncomfortable with change (3); there are numerous ethical concerns related to confidentiality, security and informed consent $(4,5)$; some supervisors feel that technology inhibits the growth process in supervisees and stunts the supervisory relationship. Bernard et al. (2) and Kanz (4) further note a lack of perceptual cues access to supervisor is not guaranteed, there is a reliance on technological skills of the supervisor and the supervisee and there are expenses of updates/new editions of technological items.

Considering ethical concerns, we must acknowledge - according to Sawyer and Schechter as cited by Koocher et al. (6) - that even prior to the Internet and the World Wide Web, mental health professionals expressed concerns about the threats posed to individual privacy and confidentiality by computerized data systems. Clients' documentation often contains confidential material, and as long as their documentation exists, someone else other than the therapists themselves may access them. Huge amounts of information can now be stored in small electronic, magnetic or optical packages that can often be easily 
and quickly transmitted, misused, stolen or misplaced (6). Koocher et al. (6) also state that in this sense modern telecommunications and computers are substantially complicated matters. This is why we must first consider how to protect the confidentiality of our clients when processing, transmitting, storing and archiving their personal data (6). Regardless of the work setting or the developmental level of the supervisee (from a beginner student to a professional psychotherapist), supervisors have two overarching ethical responsibilities: to promote the professional development of supervisees and to protect the welfare of those receiving help $(2,7)$.

However, many of the ethical considerations related to the confidentiality of client information and to how the use of technology might influence the training process, supervisory relationships, and supervisee development have not changed at all. As we all know, ethics is a philosophical discipline that defines the understanding of moral judgement and action (8). Ethics refers to standards of conduct that are established by professional organizations in reference to professional behaviour, and represents the ideal standards expected by the profession. The use of technology in supervision (TAS - technology assisted supervision) is a relatively new practice and complete guidelines have not been developed yet (9).

A review of literature by Rousmaniere (1) revealed a range of ethical issues in using technology-assisted supervision (and training), which can be grouped into three categories: the supervision process, legal and regulatory issues and technology. Supervision process issues are methods of communication between the supervisor and supervisee, the evaluation of a trainee, collaboration and the supervisory working alliance, procedures for when a trainee is judged not competent, local backup supervisors (if using distance supervision), the cultural competence of the supervisor and supervisee and procedures for the termination of supervision. Legal and regulatory issues concern local laws and regulations that apply to the supervisor's and supervisee's locations, plans for client or supervisee emergencies, informed-consent procedures for both client and supervisee and appropriate liability insurance. Technological issues are considered backup procedures for cases of technological failure, how and where data will be stored, backed up and deleted, rules for privacy, security and confidentiality of both the client's and the supervisee's information, software security standards for all computers (e.g. antivirus software), standards regarding the use of mobile devices, social software, cloud computing, training and competency standards.

The literature $(1,9)$ puts the biggest emphasis regarding ethical issues and TAS on the importance of the supervisory working relationship and questions of processes, informed consent, the evalu- 
ation of supervisees' efficacy and confidentiality. These are described in more detail later in the article.

\section{SUPERVISORY WORKING RELATIONSHIP AND QUESTIONS OF PROCESS}

Effective supervision involves the formation of a collaborative relationship in which supervisees feel safe enough to explore and disclose how they conceptualize and work with clients, how their own cultural background and personal experiences may influence their professional work and when they may be struggling with specific issues or clients. In the supervision literature, the importance of the working alliance in the supervisory process has been widely stressed (10), especially the responsibility of supervisors to foster such relationships, which includes the ethical principle of beneficence, promoting the growth, well-being and effectiveness of supervisees and indirectly preventing harm of clients or non-maleficence. Furthermore, supervisors try to form collaborative, respectful and meaningful relationships in which they recognize and accept supervisees' emotions (e.g. embarrassment over what supervisees consider to be an ineffective or inadequate session). Such relationships allow supervisors to pay attention to possible problems that can interfere with the professional development of supervisees and/or client growth and well-being (10).
An important concern in the use of TAS is how it may impact the formation and maintenance of a supervisory working alliance, especially a potential lack of nonverbal communication cues. Racial, gender and cultural differences could also cause misunderstanding $(1,5,11)$. Therefore, it is important for supervisors using TAS to pay attention to the factors that influence the working alliance in general (1), such as developing a collaborative relationship, being able to attend to nonverbal communication and cultural issues and ensuring that supervisees have a clear understanding of what to expect from the supervisor and the supervisory process.

One way to facilitate the development of a strong supervisory working alliance is for supervisors to engage in sound and informed consent practices throughout the process (9).

\section{INFORMED CONSENT}

Through the 19th and into the 20th century, the sharing of information, collaboration or patient involvement in decision making was mostly uncommon, and as a result, patients were more vulnerable to abuse or harm. Many of the currently accepted features of informed consent seen in the APA's Ethical Principles of Psychologists and Code of Conduct (12) reflect the chronological development, defined through numerous case laws. Although informed consent 
has its origins in medicine and it originally concerned specific discrete medical procedures, it has, over the years, become an essential aspect of each client's participation in the psychotherapy process (13).

Informed consent is a legal and ethical concept directed at the patient's consent for suggested treatment or prospective therapeutic relationship (14). The concept of informed consent originated from the recognition that individuals have the rights to freedom, autonomy and human dignity. These rights cannot be denied due to clients' mental health status or condition (15). Informed consent is not a singular event, but rather an ongoing process (13) used to inform, protect and empower clients (9), but also to protect the therapist (15). Informed consent has become a fundamental building block of the therapeutic relationship (16).

Ethically, consent must be truly informed (the prospective client is provided with enough information to make a reasonable decision) and voluntary (not forced or coerced), and the individual must be competent to make a decision (17). If, after reviewing informed consent materials, prospective clients are unwilling to participate, they must be free to decline without undue influence or coercion and may choose to seek services from another health care professional (16). As circumstances or situations change in the supervisory relationship (e.g. changes in the supervisee's responsibilities), the informed consent agreement should be updated (18).

There are three levels of informed consent to supervision: 1 . clients must consent to treatment with the supervisee; 2. clients must do so with the understanding that their case will be supervised; 3 . supervisees consent to supervision (19). Falvey (20) added "supervisee consent to supervision with a given supervisor" (p.71) and "institutional consent to comply with the clinical, ethical and legal parameters of supervision for the discipline(s) involved" (p.71).

\section{Informed consent between supervisor and supervisee}

Informed consent is an ongoing process that begins when supervision is first arranged and continues up to the termination of the supervisory relationship. Supervisors should engage in informed consent practices and provide supervisees with written documents (via e-mail or in person) and verbally review these with supervisees. Ethically sound informed consent practices also require some form of dialogue between the supervisor and supervisee - they should establish whether the supervisors and supervisees have a shared vision of the process of supervision and of their responsibilities in that process, preventing potential issues that may arise either within the supervisory dyad or between supervisees and clients. Bringing the supervisee into the conversation as a 
partner, rather than dictating all of the policies to the supervisee, helps build autonomy, as the supervisee is able to ask questions and possibly negotiate some terms of supervision (e.g. time of the meeting). Moreover, engaging in strong supervisory informed consent practices promotes the principles of fidelity and veracity, as both the supervisor and supervisee develop a clear understanding of their expectations for the supervisory process, the role of the supervisee and the role of the supervisor. The supervisor also uses informed consent conversations to assess the emotional and learning needs of the supervisees and determine the best way to structure supervision modelling beneficence. Having all of these factors in place facilitates the principle of veracity or being truthful within the supervisory relationship. It also allows supervisors to assess the understanding of supervisees regarding all aspects of the supervisory process, including how the use of technology may influence that process. These are concepts of effective supervision, with or without technology, but they are especially important when utilizing online supervision, as communication misunderstandings may be more likely to occur $(1,5)$. Powell and Migdole, as cited by Glossof et al. (9), also consider this.

An informed consent agreement includes the following topics: expectations, responsibilities and obligations of both supervisor and supervisee, fees and financial arrangements relevant to the supervisory relationship; scheduling and emergency contact information; documentation and record keeping requirements; the use of any audio and video recordings; evaluation and feedback to include the expectations and requirements for successful completion of the training experience; expectations regarding confidentiality and any reasonably anticipated limits to confidentiality; legal requirements such as mandatory reporting requirements; expectations regarding the availability of the supervisor and when the supervisee should contact him or her; and information about how and when the supervisory relationship will end (2,16,21-23).

\section{Informed consent between supervisee and client}

Zur (15) explains the process of securing informed consent in three phases: 1. Providing the client with information. As soon as possible in the process of the development of the therapeutic relationship, the therapist discusses with the client the nature, anticipated course and the most likely outcomes of the therapy, the potential risks and benefits of the therapy and feasible alternatives to the treatment (emphasis on the element of choice), the fact that their decision is given voluntarily and that they have a right to refuse the treatment, the principle of confidentiality and its limits (the client is informed about storing, processing and 
transmitting personal data, e.g. communicating by email, recording a session), the engagement of a third person (when the therapist is a trainee, the client is informed about the therapist's training and need for supervision, the client is given the name of the supervisor) and payment. At this stage, the client is encouraged to ask questions and to be engaged in a dialogue or discussion with the therapist. Information should be presented in a clear and comprehensible way $(15,24)$. 2. Evaluating the client's capacity to understand the information. The therapist first evaluates the client's capacity to understand the information and to make an informed decision regarding the involvement in therapy. The next step for the therapist is to check whether the information provided was clearly understood by the client (15). The therapist should be attentive to the potential impact of language, age, developmental level, cultural background and other factors that might affect the informed consent process. Dilemmas exist regarding competence, understanding and voluntariness to obtain consent from minors, inpatients, prisoners, those with cognitive impairment, etc. (13). The therapist is particularly responsible for the welfare of those clients that are not able to engage in the process of informed consent or when informed consent is gained with limitations due to the client's limitations (chronological or mental age, mental health and limitations due to the forensic process) (25). It is a challenge to understand when to share information, how much information to share and when this process may be counterproductive (13). 3. Obtaining consent from the client. The client acknowledges that they have been informed and expresses their consent in some way. By obtaining informed consent, the therapist possesses proof of consent, which becomes even clearer in the case of informed consent in writing (15). It helps to ensure that clients understand what they are agreeing to, provides them with a written document they may refer to and review over the course of the professional relationship and it allows the therapist to refer to it as well in the case of possible confusion or misunderstandings later (13). Obtaining a written informed consent is also important because of the new principle, called accountability, defined in the new EU Regulation on the protection of personal data (26). It means data controllers should not only apply the principles in law but they also have to be able to prove that they are accountable and that they respect the above principles. Thus, the burden of proof is with them (27). That means that a therapist (supervisee) has to demonstrate that a client gave their consent. This is usually easier when consent is obtained in writing. However, written consent is neither always possible nor always clinically advised, as Zur (15) points out. There are situations where informed consent is either not required or impossible to obtain. Other situations may avail verbal or other forms of consent but not written ones. Zur (15) warns that neither explicit nor written consent necessarily guarantees 
that consent is informed or valid. Implicit or implied consent is often referred to as consent that is inferred from a client's actions. Implied consent is given when clients engage in behaviours that reasonable people would interpret as informed choice (15). Still, the degree of implicit consent is not clear. In such cases, courts and professional bodies resolve possible issues individually. Some professional organizations' codes of ethics, such as that of the American Psychological Association $(12,24)$, do not always mandate that informed consent is written and signed (15). There are exceptions to the process of securing informed consent in psychotherapy: a therapist can release information and provide treatment and assessment in the case of a client's danger to self or others, child abuse or neglect and reasonable probability of general danger without informed consent (28).

It is the supervisor's responsibility to clarify to the supervisee the specific information that should be shared with the client. To ensure that supervisees can communicate to their clients any technology they will use during the therapy and supervisory processes, supervisors can facilitate the implementation of the supervisees' informed consent process between a supervisee and their client by using role-play or the Socratic method (9).

With TAS, including information about technology that will be used in supervision is critical to fulfilling the conditions of beneficence and to preventing harm to clients. Concretely, an explanation of audio or video recording, and how the recordings and any other information about clients are going to be transported, uploaded or shared with the supervisor (and other supervisees in case of group supervision), should be included in both written documents and verbal communication during the informed consent process with clients (9). "For example, after hearing about the various forms of technology involved in communicating with a supervisor, a client may give the counsellor permission to record counselling sessions but deny permission for the counsellor and supervisor to view those sessions on a cloud server. Given the frequency of data breaches in the media recently, therapists (supervisees) and supervisors should never guarantee that the client's information will be completely safe. Because it is not within the counsellor's power to honour the commitment (the principle of fidelity) that there will never be a breach of confidentiality when technology is involved. Making such a guarantee shows a lack of discernment" (9, p.40-41).

\section{ASSESSMENT OF SUPERVISEES' EFFECTIVENESS}

Assessing a supervisee's effectiveness through the use of distance supervision is similar to assessing a supervisee's effectiveness in traditional clinical supervision, because best practices continue to be the same regardless of the mode 
of supervision. Supervisors use methods of direct observation of the supervisees' work with clients, ideally reviewing a representative sample of a range of work with a range of clients, rather than relying only on supervisee's self-report. Watching or listening to client sessions in their entirety through live viewing or taped sessions continues to be the best way to determine effectiveness and competence. Recent technological advances may be helpful in this area, allowing for more options of live supervision, watching or listening to recorded sessions together. Distance supervision can now be equivalent to traditional supervision and can be used to enhance in-person supervision. Traditional group supervision of presenting cases and listening to or watching taped client sessions can be replicated at a distance through videoconference and simultaneous file sharing with the help of online cloud storage, video tagging and online discussions. Integrating a variety of technologies into supervision provides an opportunity to increase supervisees' competence in areas too remote for in-person supervision which, in turn, can increase the availability of services to clients, creating equality of access for underserved populations (9).

\section{CLIENT'S CONFIDENTIALITY, CONFIDENTIALITY IN A SUPERVISORY RELATIONSHIP}

Miller et al. (29) investigated patients' expectations of psychologists' duties re- garding confidentiality in psychotherapy. They found that $69 \%$ of patients believed and expected that all information shared in psychotherapy would be kept confidential, and $74 \%$ stated that there should be no exception to this rule. Finally, 96\% expressed wanting to know about any possible limits to confidentiality prior to beginning the psychotherapy relationship. Moreover, in the study by Sullivan et al. (30), it turned out that clients rate those psychologists who engage in an informed-consent process and give them detailed information at the outset of the professional relationship as more expert and trustworthy than those who do not (30).

According to Epstein, Steingarten, Weinstein and Nashel, as cited by Koocher et al. (6), trust developed through the assurance of confidentiality seems so essential that therapy might lack effectiveness without it. Even only the possibility of revealing such sensitive information outside the therapeutic relationship could be an obstacle for the development of a trustful relationship needed for successful treatment. Maintaining clients' confidentiality and welfare is therefore of primary importance to psychotherapists and supervisors and a focus of professional ethics codes and laws (9). The confidential relationship between mental health professionals and their clients is at the core of the helping relationship (6).

Supervisors are trained in maintaining client confidentiality and welfare 
when supervising in person, and new technologies add a layer of complexity to maintaining client and supervisee confidentiality. It is therefore important for supervisors to regularly update their knowledge of technology, so they can be updated about possible ways in which confidentiality may be breached and information inadvertently released or compromised (for example, having simple passwords for protected information). Using self-encrypting drives, password managers and two-factor authentication can reduce the risk of violating confidentiality. Although some violations of confidentiality are due to technological problems, other violations of confidentiality are due to human error (9).

Any time client information is not on paper and on site, supervisees and supervisors can make decisions that accidentally expose that information. The same steps that are taken to ensure supervisee confidentiality should be taken to ensure client confidentiality. Supervisors and supervisees should be cognizant of their environment and of who might overhear or see the clients' or supervisees' files. Thus, sessions need to be listened to and viewed in a closed, private environment with headphones. Just using headphones alone does not ensure client confidentiality when someone has the ability to view the video. Discussions about clients should always be conducted in a private, closed environment, regardless of whether these discussions occur in person or at a distance. Phone calls with supervisees should not occur in public places (9). Supervisors should develop clear procedures for the use of technology, for example how and where data will be stored, backed up and deleted, and procedures for use in case of technological failure (4).

Breaches in client confidentiality are also breaches of fidelity and can impact a client's trust in his or her psychotherapist or in the entire process of psychotherapy. At a minimum, the psychotherapist-client relationship is impacted, and the client may feel betrayed, which may trigger strong reactions, stress, anxiety, sadness, etc. If a client's information is inadvertently sent to the wrong e-mail, the client could be harmed significantly. It is important to determine what safety measures have been put in place to ensure that supervisees have access to direct supervision if any concerns arise, especially if the psychotherapist needs to breach confidentiality for the protection of the client or others. A clear plan in the event of an emergency should be determined at the beginning of the supervision process as part of supervision informed consent process and continuously evaluated throughout supervision (9).

Another new major technological innovation is "social" technology. Software is social when it is designed to facilitate connections with other users and sharing of data. Some new software programs are entirely social, such as Facebook - most supervisors know to never 
post confidential information on social services like that. However, an increasing number of new devices have builtin social features that users may not be aware of. Thus, supervisors run the risk of inadvertently "sharing" confidential information if they use a device with an active social feature. For example, some smart phone operating systems have a feature that will automatically upload data to the user's cloud-based Google+ account. Unless this account is set to private, the data will be available to anyone on the Internet. If the data is labelled with a client's name, then they could be potentially found whenever someone does a Google search for that name. So, when using devices or software with social features, it is recommended that supervisors carefully check the privacy settings (1).

The supervisor, in collaboration with the supervisee, will maintain a record of clinical supervision hours that includes (31) the date of the supervision session, supervision start and stop times, the modality of supervision provided, such as direct (live) observation, co-therapy, audio and video recordings and live supervision, documentation of all written communication during the supervisory relationship, such as chat histories, texts, instant messages, emails and notes on recommendations or interventions suggested during the supervision.

Some literature (31) suggests that supervisors and supervisees will negotiate an explicit, written contract outlining the following: full contact information of the supervisor, including telephone numbers, fax number, email and address; the listing of degrees, credentials, and licenses held by the supervisor; general areas of competence for which the supervisor can provide supervision (e.g. addictions, school, career, distance services); a statement documenting training in supervision and experience in providing supervision; a general statement addressing the model of or approach to supervision, including the role and responsibilities of the supervisor, the objectives and goals of supervision, and modalities (e.g. recordings, live observation); a description of the specific evaluation criteria and the formative and summative evaluation process that will be used throughout the supervisory relationship; a statement defining the limits and scope of confidentiality and privileged communication within the supervisory relationship; a fee schedule and payment arrangements if applicable; the emergency contact information for the supervisor and a list of back up supervisors local to the supervisee; disclosure, confidentiality breaches, security, and encryption policies; frequency of supervision; dates for review of the contract, how to address conflict and what the grievance process is for issues that cannot be resolved.

Koocher et al. (6) offer a special set of recommendations regarding electronic records and confidentiality issues in Textbox 1. 
Textbox 1. Recommendations regarding electronic records and confidentiality issues (6).

- Use encryption software to protect data transmission, to protect stored data with complex passwords (i.e. letters, numerals, symbols and/or upper- and lower-case mixes) and Internet firewalls.

- Discuss and develop security measures with professional and support personnel when storing files via a common server or backing them up on an institutional system or hub.

- Participate in creating appropriate role-segregation policies for mental health records when working at agencies that employ interoperable electronic health records.

- Advise both trainees and supervisors on appropriate procedures to protect client confidentiality when using the Internet for supervisory purposes.

- Keep removable data storage media in secure locations or use complex passwords to encrypt them.

- If using audio or visual records of client interactions for teaching purposes, consider distorting the client's voice or masking faces.

- Destroy recordings when no longer needed as long as their destruction does not conflict with other ethical or legal obligations to maintain scientific or professional records.

- Do not share passwords with others and do change them often.

- Remain mindful of security hazards of wireless devices and alert clients when using such devices.

- Avoid use of confidential information in email or instant messaging unless you use encryption.

- Take extra measures to protect the physical security of portable devices (e.g. laptops or smaller computers, personal digital assistants and smart phones) used to work with client data, whether used in the office or at home.

- Use privacy screens to shield monitors or other types of screens from viewing by others.

- Update virus protection software and other security measures frequently for both personal and work-space computing devices on which personal health information is kept.

- Remove all data when disposing or recycling old computers. This may require assistance from technical experts. Even after erasing files or reformatting disks, traces of data may remain. 


\section{CONCLUSION}

Modern technology enables supervision in remote geographical areas and access to supervisors otherwise not available to supervisees. On the one hand, the virtual world brings them closer together than ever before, which is true for clients as well. On the other hand, they may never meet in person and therefore miss the exclusivity of face-to-face interaction. Regarding the use of technology in supervision, there are numerous ethical concerns, especially those related to confidentiality, security and informed consent. Following professional associations' codes of ethics and legal regulations, the supervisor and supervisee should first consider how to protect the confidentiality of clients when processing, transmitting, storing and archiving their per- sonal data (6). The supervision informed consent process should also include information on all technology used, including steps taken to protect the client and supervisee (9). Although the possibilities of using technology have developed enormously over the past two decades, the standards of best practice for psychotherapy and supervision remain the same (9). To conclude, technology will continue to evolve, but the ethical principles remain constant: mental health professionals are ultimately responsible for safeguarding the privacy of material entrusted to them in confidence (6). Regardless of the type of technology used, supervision is supervision: it should be equally effective in person and online, follow best practices and enhance the psychotherapy process (9).

\section{REFERENCES}

1. Rousmaniere T. Using technology to enhance clinical supervision and training. In: Watkins CE Jr. and Milne DL, eds. The Wiley international handbook of clinical supervision. New York: Wiley; 2014. p. 204-237.

2. Bernard JM and Goodyear RK. Fundamentals of Clinical Supervision (5th ed.). Boston: Pearson Education, Inc:; 2014.

3. Byrne A and Hartley M. Digital technology in the 21st century: Considerations for clinical supervision in rehabilitation education. Rehabilitation Education. 2010; 24, 57-68.

4. Kanz JE. Clinical-supervision.com: Issues in provision of online supervision. Professional Psychology: Research and Practice. 2001; 32(4), 415-420.

5. Vaccaro N and Lambie GW. Computer-based supervision: Ethical and practical implications for counsellor educators and supervisors. Counsellor Education and Supervision. 2007; 47, 46-57.

6. Koocher GP and Keith-Spiegel P. Necessary Secrets: Ethical Dilemmas Involving Confidentiality. Continuing Education Courses. 2018. Retrieved from http:/www.continuingedcourses.net/active/courses/ course094.php

7. Falender CA and Shafranske EP. Clinical supervision: A competency-based approach. Washington, DC: American Psychological Association; 2004.

8. Remley TP and Herlihy B. Ethical, legal, and professional issues in counselling. 4th ed. Upper Saddle River, London: Pearson; 2014. 
9. Glosoff HL, Renfro-Michel E and Nagarajan S. Ethical Issues Related to the Use of Technology in Clinical Supevision. In: Rousmaniere T and Renfro-Michel E, eds. Using Technology to Enhance Clinical Supervision. Alexandria, VA: American Counselling Association; 2016. p. 31 - 46.

10. American Counselling Association. ACA Code of Ethics. Alexandria, VA: American Counselling Association; 2014.

11. Rousmaniere T. and Frederickson J. Internet-based one-way-mirror supervision for advanced psychotherapy training. The Clinical Supervisor. 2013; 32: 40-55

12. American Psychological Association. Ethical principles of psychologists and code of conduct. American Psychologist. 2002; 57, 1060-1073.

13. Barnett JE, Wise EH, Johnson-Greene D, Bucky SF. Informed Consent: Too Much of a Good Thing or Not Enough? Professional Psychology: Research and Practice. 2007; 38(2), 179-186.

14. Tjeltveit AC. Ethics and Values in Psychotherapy. London: Routledge; 1999

15. Zur O. Introduction to Informed Consent in Psychotherapy, Counselling and Assessment. Zur Institute: 2018. Retrieved from https://www.zurinstitute.com/informedconsent.html

16. Thomas JT. Informed Consent Through Contracting for Supervision: Minimizing Risks, Enhancing Benefits. Professional Psychology: Research and Practice. 2007; 38(3), 221-231.

17. Haas LJ and Malouf JL. Keeping up the good work: A practitioner's guide to mental health ethics (4th ed.). Sarasota, FL: Professional Resource Exchange; 2005

18. Barnett JE and Molzon CH. Clinical Supervision of Psychotherapy: Essential Ethics Issues for Supervisors and Supervisees. Journal of Clinical Psychology. 2014; 70(11), 1051-1061.

19. Bernard JM and Goodyear RK. Fundamentals of clinical supervision (2nd ed.). Boston: Allyn \& Bacon; 1998.

20. Falvey JE. Managing clinical supervision: Ethical practice and legal risk management. Pacific Grove CA: Brooks/Cole; 2002.

21. Barnett JE. Ethical issues in clinical supervision. The Clinical Psychologist. 2011; 64, 14-20.

22. Falender CA. Getting the most out of clinical supervision: A guide for practicum students and interns. Washington, DC: American Psychological Association; 2011.

23. Thomas JT. The ethics of supervision and consultation: Practical guidelines for mental health professionals. Washington, DC: American Psychological Association; 2010

24. American Psychological Association. Revision of ethical standard 3.04 of the "Ethical Principles of Psychologists and Code of Conduct" (2002, as amended 2010). American Psychologist. 2016; 71, 900

25. Slovenian Psychologists' Association. The Code of Professional Ethics of Slovenian Psychologists (Kodeks poklicne etike psihologov Slovenije); 2002.

26. EU Regulation on the protection of personal data (2016/679). Retrieved from http://eur-lex.europa.eu/ legal-content/EN/TXT/?uri=OJ\%3AL\%3A2016\%3A119\%3ATOC

27. European Patients' Forum. The new EU Regulation on the protection of personal data: what does it mean for patients? A guide for patients and patients' organisations; 2016

28. Slovenian Psychologists' Association. The Code of Professional Ethics of Psychologists' (Kodeks poklicne etike psihologov); 2018.

29. Miller DJ and Thelen MH. Knowledge and beliefs about confidentiality in psychotherapy. Professional Psychology: Research and Practice. 1986; 17, 15-19

30. Sullivan T, Martin WL and Handelsman MM. Practical benefits of an informed-consent procedure: An empirical investigation. Professional Psychology: Research and Practice. 1993; 24, 160-163.

31. Stretch LS, Nagel DM and KA. Ethical Framework for the Use of Technology in Supervision. Therapeutic Innovations in Light of Technology. 2012; 3(2), 37-45. 